\title{
Evidence-Based Practice: Developing a Trauma-Informed Lens to Case Management for Victims of Human Trafficking
}

\author{
Kristin Heffernan • Betty Blythe
}

Published online: 1 April 2014

(C) Springer International Publishing 2014

\begin{abstract}
The purpose of this study was to evaluate program adherence, process factors, and success of human trafficking case management services in an agency providing services to international human trafficking victims as there is a lack of research on the effectiveness of services for this population; however, using a trauma-informed lens has been suggested by other researchers (see Mary \& Johns, 2011). As such, this paper describes services provided to international victims of human trafficking in an upstate New York facility and outlines the use of a trauma-informed lens in conjunction with a clientcentered approach to delivering case management to persons who have experienced human trafficking. External evaluators were used to conduct a process evaluation of the case management services in a single agency. Evaluation of adherence to the program's service protocol used a deductive approach to identify if the service providers (case managers and program director) followed the seven principles of trauma-informed care (TIC) in their practice. Modes of examining for TIC indicators included participant observation, content analysis of case folders, and other paperwork utilized within the agency as well as conversations with case managers and the program director. Findings indicate that a trauma-informed lens was successfully implemented, and the small-scale preliminary feedback from clients $(N=7)$ is that they feel safe, respected, and confident to live independently in an environment of their choosing.
\end{abstract}

Keywords Human trafficking · Case management . Trauma-informe- care

K. Heffernan $(\bowtie)$

The College at Brockport, SUNY, New York, USA

e-mail: kheffern@brockport.edu

B. Blythe

Boston College, Boston, MA, USA

e-mail: blythe@bc.edu

\section{Human Trafficking}

Human trafficking is a horrific crime against humanity, often bringing about both physical and emotional trauma to its victims. The Victims of Trafficking and Violence Protection Act of 2000 (TVPA) defines human trafficking as:

(a) "Sex trafficking in which a commercial sex act is induced by force, fraud or coercion, or in which the person induced to perform such an act has not attained 18 years of age." (hence, all children engaged in sex exploitation are seen as victims under federal law); or

(b) "The recruitment, harboring, transportation, provision or obtaining of a person for labor or services, through the use of force, fraud, or coercion for the purpose of subjection to involuntary servitude, peonage, debt bondage, or slavery." (U.S. Department of State, 2007, p. 7).

It is difficult to obtain exact numbers of how many persons are trafficked into the USA due to the insidious nature of human trafficking; however it has been projected that somewhere close to 14,500 and 17,500 individuals get trafficked annually (Clawson et al., 2009). The same holds true for the estimation of US domestic trafficking numbers. Estes and Weiner (2001) is one of the only studies to date reporting estimated numbers of American youth at risk for sex trafficking, placing the number between 244,000 and 325,000 with another 199,000 estimated incidents of sexual exploitation of minors occurring each year in the USA.

The USA is both a source of transit and destination for men, women, transgender individuals, and children, trafficking both US citizens as well as foreign nationals (Macy \& Johns, 2011; Srikantiah, 2007). Risk factors for becoming trafficked include but are not limited to: living in poverty, young age, limited education, lack of work opportunities in home land or local country, and lack of family and other 
supports (Clawson et al., 2009). Persons who are trafficked can be subjected to forced labor, debt bondage, involuntary servitude, and/or sex trafficking, which can occur in many licit and illicit industries or markets (Kim \& Hreshchyshyn, 2004; Macy \& Johns, 2011). Again, these include, but are not limited to, brothels, massage parlors, street prostitution, hotel services, hospitality, agriculture, manufacturing, janitorial services, construction, health and elder care, and domestic service (as cited in Dowling, Moreton, \& Wright, 2007; Patel, 2011).

Very few studies have explored the aftercare service needs for persons who have been trafficked. To date, the most complete review of the literature regarding aftercare services indicated that a comprehensive continuum of care services is needed which includes "(a) immediate and crisis needs, (b) ongoing needs, and (c) long-terms needs. The review also identified seven core service areas within this continuum: (a) basic necessities; (b) secure, safe shelter, and housing; (c) physical health care; (d) mental health care; (e) legal and immigration advocacy; (f) job and life skills training; and (g) substance abuse services" (Macy \& Johns, 2011, p. 95). While, research on the effectiveness of these services is still woefully lacking, Macy and Johns (2011) were able to create an aftercare delivery framework model based on their review which included using a trauma-informed care focus. If we can agree that the experience of being trafficked is in and of itself a traumatic experience, then using a trauma-informed care (TIC) framework for providing services to traumatized individuals makes sense.

\section{Effects of Human Trafficking}

According to Tsutsumi, Izutsu, Poudyal, Kato, and Maru (2008), trafficked individuals experience trauma by the very nature of being trafficked. Exposure to chronic trauma often causes post-traumatic stress disorder (Courtois, 2004; Flowers, 2001; Raymond \& Hughes, 2001; United States Department of State [US DOS], 2006). In many human trafficking cases, the perpetrators induce psychological trauma by destroying the victim's sense of self in relation to others. Traffickers often terrorize their victims through threats of death and serious harm against both the victims and their families (Kim \& Hreshchyshyn, 2004; Patel, 2011; Shigekane, 2007). They may threaten them by telling them that they will be deported or arrested if they seek help from the police or others (Hopper, 2004). In addition to emotional abuse, traffickers may create alcohol or drug dependencies to further entrap victims (Raymond \& Hughes, 2001). Such techniques often leave victims feeling helpless and hopeless, so even if they come in contact with law enforcement or social services, they are hesitant to ask for help. This, in part, is a natural result of the psychological coercion they experience, but also because they fear retribution from the trafficker
(United States Department of Justice [US DOJ], 2006). Additionally, physical health is at stake for those being trafficked.

Traffickers may start out by sexually assaulting or committing other physical abuse against their victims (Tsutsumi, Izutsu, Poudyal, Kato, \& Maru, 2008). Often, perpetrators bring about terror through inconsistent and unpredictable outbursts of violence towards their victims (Shigekane, 2007). Such violence can result in fractures; complications related to forced abortions, gastrointestinal problems, and unhealthy weight loss, ultimately, can lead to alcoholism and drug addiction (US DOS, 2006; Zimmerman et al., 2008). It is not uncommon for perpetrators to employ a combination of abusive methods, including torture, to establish and maintain control over their victims (Perilla, 1999).

\section{Developing Trauma-Informed Case Management}

In the project reviewed, the agency recognized that each victim interprets and creates her or his own reality of human trafficking. As such, the clients were the ones who labeled themselves as being labor-trafficked, sex-trafficked, or both. Moreover, the agency staff went into this project acknowledging that the emotions felt by each client, along with the client's cultural responses to her or his experiences, may be different for each victim or survivor of human trafficking.

This realization motivated the program staff to attempt to identify the best methods of providing case management to victims of human trafficking, one that allowed clients to continue to voice their experiences through their own unique perspectives in a nonjudgmental atmosphere. Program staff also recognized that, regardless of the type of human trafficking experienced by the individual, the experience of human trafficking in and of itself is traumatic. This led them to develop a TIC service protocol for case management. This meant looking at their current method of providing case management (a client-centered approach) and designing case management techniques, goals, and policies that directly address the impact of trauma on their clients in order to facilitate recovery.

While there may not be a consensus as to what constitutes the components of TIC and as such no standardized definition exists as of yet, it is widely accepted that TIC refers to a philosophy or cultural point of view integrating awareness and understanding of trauma (Hopper, Bassuk, \& Olivet, 2010). "TIC is an overarching framework that emphasizes the impact of trauma and guides the general organization and behavior of an entire system" (Hopper et al., 2010, p. 132). A further review of the literature identified the following key themes as central to TIC: trauma awareness, safety, rebuilding control, and using a strength-based approach (Hopper et al., 2010, pp. 81-82). The agency under review 
used the first three of these concepts and then further identified proponents of a strength-based approach in order to develop their TIC case management philosophy. Hence, in dissecting what is meant by a strength approach, we can see how the other principles used in their TIC case management have been developed.

A strength-based approach views all persons via their "capacities, talents, competencies, possibilities, vision, values and hopes" (Sallebey, 1996, p. 297). The perspective takes into account the whole human being which means that attributes such as age, culture, ethnicity, and the like... are viewed as strengths. In this way, cultural competency of the practitioner is essential in using a TIC perspective. Collaborating with the client also is critical. The social worker should encourage client participation, seeking their opinions and input. This approach posits that strengths, rather than deficits or "problems" of people and their environment, should be the focus of the helping process. Accordingly, human beings have the potential for growth and development and the ability to identify their own needs (Fast \& Chapin, 1997) and practitioners need to empower clients to be able use their own voice to express what they need.

\section{The Importance of Evaluation}

Process evaluation is a critical step when assessing what works with regard to service provision within an organization. It often includes a systematic evaluation of the process and outcomes of a program (Zakrzewski, Steven, \& Ricketts, 2009). Given that developing best practice in delivering services to persons who have been trafficked is still in its infancy, monitoring and evaluation research should be a part of every agency's program. More specifically, external evaluation can help discover "best practice" approaches (Gozdiak \& Bump, 2008). Additionally, developing case management methods that have proven effectiveness aids social workers in protecting their clients and their credibility while adhering to the social work profession's code of ethics.

One area of focus within process evaluation is exploration of how a program is actually delivered (Domitrovich \& Greenberg, 2000). The process evaluation reported here centers on the concepts of program adherence, process factors, and success based on feedback from clients. Program adherence involves making an objective estimate of the percentage of the protocol that is implemented in actual service delivery, known as implementation fidelity (Law \& Shek, 2011). Practice effectiveness or success refers to the extent to which the services provided yield their intended results (Cournoyer, 2004). In this case, the goal is that persons receiving services will gain autonomy from their trafficker and feel secure to return to independent living in the community of their choice.

\section{The Program Under Evaluation}

The program being evaluated was established in partnership with a legal service in New York which provides legal aid to victims of human trafficking. The agency itself is located in upstate New York and is funded by the Office of Victims of Crime. Its remit is to provide services to certified victims of human trafficking via case management in order to help the survivor of trafficking become independent of his or her trafficker and to live self-sufficiently. For the purpose of ensuring anonymity for survivors of human trafficking that utilized this agency, the name of the agency will not be revealed in this study. Case managers are responsible for coordinating needed services within the community. The services offered by the workers include in-house services (e.g., crises intervention, emotional support-non-mental health counseling, explanation of legal rights and protections, interpreter/translator, protection/safety planning, social service advocacy and explanation of benefit entitlements and their availability, transportation, and training on human trafficking as a traumatic event to clients and other agencies) and referral services (e.g., child care, clothing/food, dental, employment assistance, legal services-including immigration advocacy, literacy education and or on the job training, medical emergency and long-term mental health services, repatriation, and shelter/housing). The director of the program is a licensed social worker (LMSW) in NY and is trained in TIC. One of the case workers has her Masters in Social Work (MSW) while the other one has an undergraduate degree in a related field, but will be returning to a university to get her MSW

\section{Methods}

The program evaluation studied the delivery of case management services for international victims of human trafficking to explore whether or not the services being offered were adhering to a trauma-informed lens of delivering case management services. The agency's protocol for service delivery combined proponents of traumatology and a strength perspective to a client-centered approach and developed seven TIC principles which are to be interwoven into the agency's case management practice. The TIC principles include the following: (1) recognize, the impact of the trauma of human trafficking on the clients' development and coping strategies by validating these experiences throughout the helping process; (2) safety, ensuring physical and emotional safety; (3) trustworthiness, maximizing trustworthiness through task clarity and maintaining appropriate boundaries; (4) choice and control, prioritizing survivor choice and control; (5) collaboration, maximizing collaboration and sharing power with survivors; (6) empowerment, prioritizing survivor empowerment and skill 
building; and (7) language access and cultural competency, eliminating service disparities based on language or culture.

Evaluation of the adherence to the TIC principles used qualitative, deductive content analysis, exploring the use of the seven principles of TIC as themes in both the documented forms as well as the verbal exchanges used in practice by the director of the program and the case managers. The documentation reviewed included forms handed out to clients or used to work with clients as well as client case notes. Additionally, the language used while in meeting with clients, in case meetings, and in staff meetings was assessed.

In reviewing the case files and other forms, the researchers looked for specific TIC terms (e.g., safety, collaboration) as well as descriptions of the terms. When the researchers inferred the meaning, they then spoke with the case managers or program director to understand the meaning of the written material. This was especially important during the observation stage and when analyzing the client folders.

Participant observation was used to evaluate the language used in both client case meetings as well as regular staff meetings. In order to investigate the observed data in terms of constructed meanings, it was pertinent to include the feedback and opinions of the observed participants (Creswell, 2013). So, for example, during the observation stage, the researcher would write down there observations and would then ask the research participant about what was observed. As such, the findings of this study reflect participant observation of interactions between case managers, case managers and clients, and case managers and the program director. Additionally, one case management meeting and two regular staff meetings were observed. Finally, feedback from clients who left the program to live independently within the community of his or her choice was gathered using an exit interview.

The client interview was the last step of the data collection. So, those clients transitioning out of services were interviewed in order to gain their perspective of the effectiveness of the case management provided. Hence, the process evaluation involved multiple methods of data collection.

In order to measure the success of the case management services, we used one of the program goals: Clients will be able to feel safe to live independently within an environment of his or her choice. We then constructed exit interview questions around the TIC principles and the persons' perception of being ready to leave services and live independently. We were only able to interview seven clients because only seven clients, over the period under review, had transitioned into independent living. While this number may seem small, it is important to remember that even this small number of responses is important due to the fact that so little is known about effective services for persons that have been trafficked

Additionally, it is important to state here that due to the large quantity of qualitative data collected, it is impossible to demonstrate each TIC principle in action, so the findings are more of a snapshot of how we determined if they were in place in the case managers' practice. While content analysis can be quantified by counting the number of times a particular category or concept appears in practice, as researchers, we felt that such reporting lessened the value of the research, taking away from the richness of the actual and interpreted content.

\section{Findings}

\section{Demographics}

Between October 2010 and October 2011, 22 individuals were referred to the agency as possible victims of human trafficking. Of these referrals, 12 of the clients accepted services from the agency. There were seven female clients, three of whom identified as survivors of sex trafficking, two identified as labor trafficking survivors, one identified as both, and one did not categorize herself other than specifying that she was a survivor of human trafficking. The five males who accepted services all identified as labor trafficking survivors. All 12 case files were reviewed, and at the time of this paper, 7 clients have been interviewed ending services, as the rest are still receiving services. All of the clients referred for services were trafficked from other countries into the USA. Clients referred to services represented 13 different geographical locations, but the majority of clients were from Mexico.

\section{Case Management Observations}

We observed the engagement, assessment, planning, implementation, and evaluation of services as the case managers and clients interacted, specifically looking for the enactment of trauma-informed concepts in practice. We found the TIC principles of safety, encouraging the client to make his or her own choices, working in collaboration with the client, attempts to build a trusting relationship, and the principle of language access and cultural competency being enacted in case management practice via our observations.

For example, during the engagement and assessment stage, case managers helped provide emotional safety by orienting clients to the agency by means of an agency tour, introducing them to everyone in the agency and showing them the location of building exits and entrances. When we asked about this practice, it was explained that this was done to help the client feel safe in his or her environment and because if they know who does what, they can better make requests for services themselves.

Case workers also conducted safety plans at every meeting we observed. They started meetings, by asking if the client felt safe to talk with them and then proceeded to go over the safety plan to make sure that it was still applicable. Also, the case 
managers often reviewed their role, the role of the interpreter and their commitment to confidentially. Again, when asked about these practices, we were told that they do these things to help the clients feel safe both physically and emotionally. Furthermore, the staff went on to explain that they do these things at each meeting because repetition and creating clear boundaries and expectations help to build trust as well as give the clients a feeling of continuity.

Collaboration and giving the client choice were observed during the assessment and planning stages. Case managers let the clients choose the meeting time and place. They also listed the services and allowed the clients to choose services that they needed. Clients were verbally encouraged to make their own care plans. In several instances when sa client was not sure if he or she could make the appointment, they were told that they could wait and give the case manager a call back once they checked their calendars. At the close of the meeting, the case manager reviewed the care plan again with the client to make sure that they understood what the client needed. Often, an interpreter was present at the meeting demonstrating the value of language access. We also observed that the case managers began sessions by asking the client if they wanted anything to drink or eat and how they were doing. When asked about the small talk, they said they were trying to build trust with their clients.

\section{Client Case Plan Meeting Observation}

The client case planning meeting primarily focused on the case management process with the client. It was led by the program director. The program director opened the meeting by checking in with the case managers to see how they were doing, giving time for them to let off steam and make small talk. When asked about this opening, the program director stated that she tries to create a space were the case managers feel safe to open up and share what is going on in their professional and personal lives as both can be affected by working with trauma victims. This was interpreted as demonstrating both TIC principles 1 recognize and 2 safety. It was clear that the program director understood the impact of working with human trafficking victims from our discussion and, by allowing case managers to let off steam, she was intending to create an emotionally safe environment.

The director also discussed the issue of having appropriate interpreters for the client (TIC principle (7) language access and cultural competency). Mostly, the conversation was a way of checking that the system of getting an interpreter was working. The director stressed "the importance of prearranging an interpreter for the client to help them feel welcomed and understood." One case manager mentioned that a specific interpreter had cancelled again and the director said "that's a problem. I'll call and talk with ...... we're not going to use that interpreter anymore." She then went on to say that it was important that the clients have a consistent interpreter in order to help their recovery and build trust (TIC principles 3 trustworthiness, 4 choice and control, 6 empowerment).

Next, a staff member discussed a current client's safety plans. The conversation also became broader as the director initiated a dialogue about other ways the case managers made sure that their clients felt safe (TIC principle 2 safety).

During one of the case reviews, an issue came up with regard to a client who was not following through with one of the care plan goals which was to attend an English as a second language (ESL) course. The case manager stated that she had received a phone call from the ESL teacher stating that the client had not attended the last two classes. The director started by saying "it is the client's choice... if he did not want to participate in the ESL classes, then he did not have to attend." She further suggested that the case manager double check to see if this was a service that the client still wanted (TIC principles 4 and 6 choice and empowerment, respectively) .

This led to a conversation about how the case managers ensure client choice. During this discussion they examined ways to help the client understand that it is her or his choice to participate in services and how to get clients involved in service planning. In turn, this led to a discussion about how to reassure the client that it is okay to change one's mind (TIC principles 4 choice, 5 collaboration, 6 empowerment). It was decided that if the client being discussed no longer wanted to take ESL classes, he or she should be encouraged to call and withdraw from the classes (TIC principle 6 empowerment).

\section{First Staff Meeting}

The first observed staff meeting started by reviewing the newly developed and/or redesigned forms used in the case files. The first form reviewed was the Roles and Responsibilities form. When asked the purpose of this form, the director explained that it lays out the expectations for employees at the agency as well as expectations for their clients in an attempt to demonstrate collaboration and consistency. Further stating that "it is an agreement that the client and the worker both sign, once the client decides if she or he wants to receive services from the agency" (TIC principles 4 choice and collaboration and 6 empowerment). Again, the forms were reviewed specifically looking for key terms that could be interpreted as themes from the TIC principles. So, for example, the opening of the agreement reads as follows:

At Agency XYZ, "we aspire to provide a safe environment and are committed to trying to meet the needs of our clients (TIC Principle 2: Safety). All participation is voluntary and clients have the right to refuse participation in the program or any particular services they are not interested in (TIC Principles 4 and 6: Choice and Empowerment). We are here to assist you with meeting 
your needs. Either party has the right to end a session at any time they feel uncomfortable (TIC Principles 4 and 6 again). If you agree to accept services here, we have expectations for both our staff and clients that we expect you to agree to."

It then goes onto list the agency's expectations. For example:

1. "We will provide a safe, calm, professional meeting environment at Agency XYZ- we want you to always feel welcome here. (TIC principle 2 safety).

2. We will treat you with dignity and respect in an

3. environment free from discrimination (TIC principle 7 language and cultural competence).

4. We will provide all services and written materials to you in a language that you understand or provide an interpreter (TIC principle 7 language and cultural competency).

5. We will respect your unique cultural background..." (TIC principle 7 language and cultural competency).

And then, the client's role and responsibilities:

1. "I will participate in the development of and have input on any changes made to my service plan (TIC principle 4 choice, 5 collaboration, and 6 empowerment).

2. I will attend all scheduled sessions and contact staff in advance if an emergency arises and I cannot attend (TIC principle 3 trustworthiness, 6 empowerment).

3. I will respect staff and express any concerns in a calm manner, understanding that aggressive behavior will result in termination of the session.

4. I will have the opportunity to resolve all conflicts and concerns regarding services without fear of reprisal." (TIC principle 2 safety, 5 collaboration).

\section{Second Staff Meeting}

The second staff meeting that was observed started off the same as the first, but then the director switched gears to reiterate the importance of understanding human trafficking as a traumatic event (TIC principle 1 recognize). She reiterated the importance of understanding the trauma experienced by the client and the need to minimize re-victimization while facilitating not just recovery but empowerment. This led to a discussion about staff training needs. The conversation appeared to be a way of validating an understanding that the program as a whole needed to commit to understanding and integrating knowledge about violence and trauma-related incidents into their overall mission and program goals (Harris \& Fallot, 2001). This entire dialogue demonstrated TIC principle 1 recognize.
One of the team's goals is to partner with community services and to help educate them about human trafficking (TIC principles 1 recognize and 5 collaboration). Thus, the team moved to brain storming about their current community partners and identified where deficits exist. One area that was highlighted was the need to develop closer ties with local mental health care providers as their clients were waiting too long before being seen for their mental health needs.

In addition, the program director reviewed each person's vacation time and talked about the importance of self-care. The program director even asked one of the case managers to think about taking a break as she had had several difficult cases recently, and the director felt she could use some time off for herself. The director explained the importance of understanding that working with trauma victims means that workers are susceptible to vicarious trauma and to help prevent this, they continually try to normalize trauma responses via these meetings (TIC principles 1 recognize and 2 safety).

\section{Progress Notes and Other Forms}

Both the progress notes and newly designed paperwork were reviewed to establish if they were consistent with TIC principles of case management. The findings indicate that the case notes and other forms all include language that reflects TIC principles. Several themes form the principles of TIC were represented in case notes, albeit in very subtle ways. These themes include the first 6 TIC principles, recognize, safety, trustworthiness, choice, collaboration, and empowerment:

As one example from the progress notes, a case manager wrote as follows:

client was referred to Agency XYZ by blank for suspected human trafficking, client was unsure if she was in the right place. We spent our first meeting talking about our services going over the program's Participant Bill of Rights. Client stated he was in need of a shower, sleep and some new clothes. The client agreed to go to the safe house..... took him there, gave him my (business) card and told him I would check in on him in the morning. (TIC principles 3 trustworthiness and 4 choice)

In another excerpt from the progress notes, a case manager wrote as follows:

I met with XXX today to review the safety plan. XXX stated that he did not feel safe at... and wanted to move to a new location. We spent the rest of our meeting getting him into .... XXX felt good about this new plan and I am going with him to help him move his stuff to... (TIC principles 2, 3, 4, 5, and 6)

Additionally, the language of TIC also is evident in other forms used at the agency. This can be seen in the Roles and 
Responsibilities form which was already discussed as well as in the Participant Bill of Rights form which is given to each client once he or she decides to receive services from the agency. The Participant Bill of Rights reads as follows:

You have the right to put yourself first.

You have the right to be you.

You have the right to be safe.

You have the right to love and be loved.

You have the right to be treated with respect.

You have the right to be human - NOT PERFECT.

You have the right to be angry and protest if you are treated unfairly or abusively by anyone.

You have the right to your own privacy.

You have the right to earn and control your own money.

You have the right to ask questions about anything that affects your life.

You have the right to make decisions that affect you.

You have the right to your own opinions, to express them, and to be taken seriously.

You have the right to grow and change (and that includes changing your mind).

You have the right to say NO.

You have the right to make mistakes.

You have the right NOT to be responsible for other adults' problems.

You have the right not to be liked by everyone.

YOU HAVE THE RIGHT TO CONTROL YOUR

OWN LIFE AND TO CHANGE IT IF YOU ARE

NOT HAPPY WITH IT AS IT IS.

\section{Interviews with Participants}

The interviews with clients who were leaving the agency demonstrated that that these clients felt, safe, respected, and confident about their ability to return to their community of choice and live independently. Hence, the program was achieving one of its end goals for clients while using TIC case management. The following are a sample of questions and client responses from the exit interviews:

Question 2 Do you think that you were treated with respect by (case manager)?

"Always respectful...They really tended to us." (male client)

"My caseworker was very respectful. Everyone was respectful, always aware of my needs and there for me... I felt protected, safe. I am very happy. I got more than I expected." (male client)
"Very respectful. They were all great. Very helpful and respectful from top down. They helped me with everything. They are my family. The most important service is they helped me to be free... My caseworker is my family, she showed me everything....they were all respectful." (female client)

Question 10 How are you feeling about leaving Agency $\mathrm{XYZ}$ and living independently?

"I feel very confident in going out into the working world. I am ready to be independent. I do not have a lot of contacts within the community, but I feel safe. The four persons I live with understand me and are willing to help me if I need it...All of the services were most beneficial - they helped me to be able to live free." (female client)

"Yes, I am back in (home country), working independently..." (male client)

"Returning to home is good. They helped me make phone contacts in (home country) and paid for my taxi to the airport. They helped me with the transition. Being home feels good. I am not missing anything I need. They made me feel safe while I was in Agency XYZ." (male client)

\section{Discussion}

Participant observations along with content analysis of the case records and of other paperwork revealed ample evidence of the adherence of the TIC principles within the case management services offered at the agency. For example, during the client care plan meeting, the dialogue emphasized the importance of pre-arranging an interpreter for the client to help them feel welcomed and understood, of safety planning and of ensuring that the case manager empower the client by helping them realize that they have choices with regard to the services they accept. All of these components underpin the agency's TIC principles for case management.

The staff meetings also directly demonstrated these same themes. The very action taken by the staff to review the new forms demonstrated the team's commitment to adapting a trauma-informed lens. Because the forms were developed by the entire team, everyone had a chance to have input. In reviewing the forms, it appears that the staff members are committed to the principles of trauma-informed case management. The Roles and Responsibility form lays out the expected behavior as well as the rights of both client and worker. It stresses 
that the client has the choice of which services he or she wishes to participate in and lets them know that they can stop services at any time (stressing choice and empowerment). At the same time, it underscores the idea of mutual respect (creating an accepting and respecting atmosphere). For instance, it states that the client is expected to keep appointments but will attempt to call to cancel an appointment if an emergency should arise. The same themes are found in the language used to list the caseworker's roles and responsibilities.

The second staff meeting opened with the program director presenting training courses that would help her staff develop more knowledge on the topic, addressing the principle of recognizing the impact of the trauma of human trafficking on the clients' development and coping strategies by validating these experiences throughout the helping process. The priority at this point in the meeting appeared to be having the team come to an agreement that the program as a whole needed to commit to understanding and integrating knowledge about violence and trauma-related incidents into their overall mission and program goals (Harris \& Fallot, 2001). There was also a discussion of next steps for the agency. One of the team goals is to partner with community services and to educate them about human trafficking. Beyond providing better services for clients, developing new collaborations with other agencies that also deal with traumatized clients can provide emotional support for staff and reduce the sense of isolation at having to "go it alone" (Fisher, 2011).

Content analysis of the case notes and other paperwork developed using a trauma-informed lens showed adherence to the TIC principles of case management. The case management team, under their director's leadership, redesigned all of the case management paperwork in an attempt to ensure that the concepts behind TIC permeated the work that was done within the agency. In assessing the case files, several forms were developed to empower clients, while working collaboratively with them to make certain that clients felt like active participants and that they had choice and control over the services they received. The Participant Bill of Rights form is just one example of this. It includes language directly adapted from the principles guiding their case management, such as "You have the right to be safe," "You have the right to be treated with respect," and "You have the right to make decisions that affect you."

The content analysis of the progress notes revealed that case managers used the first meetings to focus on clients' immediate needs rather than completing an intake and/or gaining information regarding the client's trafficking status. This demonstrates the case manager's knowledge and understanding of the need to establish a collaborative relationship with the client. It also shows that case managers understood human trafficking as a traumatic life event that may influence the clients' recovery and coping strategies.

In the majority of the files, the case managers met with clients several times before the client felt comfortable in saying that they would like to receive services from the agency. The case managers demonstrated an understanding that the client's safety must be a priority and that trust is developed over time in order to reduce the chance of re-traumatization of the client. They created an atmosphere of respect and acceptance, so as to allow the client to voice his or her experience if she or he wished:

Finally, we reviewed other relevant written materials within the agency and found that they also reflected with the concepts of TIC. For example, the agency mission statement and the training for new employees both emphasized that they are there to provide a safe environment and are committed to trying to meet the needs of their clients.

\section{Conclusion}

While this study has its limitations (e.g., a small number of clients interviewed $N=7$ ) as there is limited research into practice methods with survivors of human trafficking, it also offers a starting point for further research on possible promising practices with human trafficking survivors. This paper presents a case management program for working with people who have been trafficked. The TIC principles underlying the program clearly recognize the significance of trauma in the response of victims and survivors of human trafficking. We would argue that any services offered to persons that have experienced human trafficking should be trauma-informed in order to reduce the likelihood or re-traumatization.

The process evaluation along with the responses from clients demonstrate early indications of intervention fidelity with regard to the TIC principles. While the initial evidence is promising, further research should examine more indicators of the effectiveness of the program with a larger sample. One thing that is clear is that many of the proponents of aftercare services found in Macy and Johns' (2011) review of after care services for victims of human trafficking hold true with regard to the current study. The case management services were comprehensive, used a trauma informed lens, and provided translation services (Macy \& Johns, 2011). Further research, however, is needed on the efficacy of service delivery for this population in or to continue to build better, more effective aftercare services.

\section{References}

Clawson, H.J., Salomon, A., Grace, L.G. (2009). Human Trafficking Into and Within the United States: A Review of the Literature, Retrieved from: http://aspe.hhs.gov/hsp/07/HumanTrafficking/LitRev/index. shtml\#Trafficking

Cournoyer, B. R. (2004). The evidence-based social work skills book. Boston: Pearson. 
Courtois, C. A. (2004). Complex trauma, complex reactions: assessment and treatment. Psychotherapy: Theory, Research, Practice, Training, $41,412-414$

Creswell, J. W. (2013). Research design: qualitative, quantitative, and mixed method approaches. Thousand Oaks: Sage.

Domitrovich, C. E., \& Greenberg, M. T. (2000). The study of implementation: current findings from effective programs that prevent mental disorders in school-aged children. Journal of Educational and Psychological Consultation, 11, 193-221.

Dowling, S., Moreton, K., Wright, L. (2007).Trafficking for the purposes of labour exploitation: a literature review. Home Office: UK, Retrieved from: http://www.homeoffice.gov.uk/rds.

Estes, R., \& Weiner, N. (2001). The commercial sexual exploitation of children in the U.S., Canada, and Mexico. Philadelphia: University of Pennsylvania.

Fast, B., \& Chapin, R. (1997). The strengths model and critical practice components. In D. Saleebey (Ed.), The strengths perspective in social work practice (2nd ed., pp. 115-131). White Plains: Longman Publishers.

Fisher, M. (2011). Factors influencing stress, burnout, and retention of secondary teachers. Current issues in education, 14, Retrieved from http://cie.asu.edu/

Flowers, R. B. (2001). The sex trade industry's worldwide exploitation of children. Annals of the American Academy of Political and Social Science, 575, 147-157.

Gozdiak, E. M., \& Bump, E. A. (2008). New immigrants, changing communities best practices for a better America. Lanham: Lexington Books.

Harris, M., \& Fallot, R. D. (2001). Using trauma theory to design service systems. San Francisco: Jossey-Bass.

Hopper, E. K. (2004). Under-identification of human trafficking victims in the United States. Journal of Social Work Research Evaluation, 5 , 125-136.

Hopper, E. K., Bassuk, E., \& Olivet, J. (2010). Shelter from the storm: trauma-informed care in homelessness services setting. The Open Health Services and Policy Journal, 2, 131-151.

Kim, K., \& Hreshchyshyn, K. (2004). Human trafficking private right of action: civil rights for trafficked persons in the United States. Hastings Women's Law Journal, 16(1), 1-36.

Law, B. M. F., \& Shek, D. T. L. (2011). Process evaluation of a positive youth development program: project P.A.T.H.S. Research on Social Work Practice, 21, 539-548.
Macy, J. M., \& Johns, N. (2011). Aftercare services for international sex trafficking survivors: informing U.S. service and program development in an emerging practice area. Trauma. Violence and Abuse, 12, 87-98.

Patel, A. (2011). Back to the drawing board: rethinking protections available to victims of trafficking. Seattle Journal for Social Justice, 9, 813-842.

Perilla, J. (1999). Domestic violence as a human rights issue: the case of immigrant Latinos. Hispanic Journal of Behavioral Sciences, 21, 107-133.

Raymond, J. G. \& Hughes, D. M. (2001). Sex trafficking of women in the United States. Report, University of Rhode Island.

Sallebey, D. (1996). The strengths perspective in social work practice: extensions and cautions. Social Work, 41, 296-305.

Shigekane, R. (2007). Rehabilitation and community integration of trafficking survivors in the United States. Human Rights Quarterly, 29, 112-136.

Srikantiah, J. (2007). Perfect victim and real survivors: The iconic victim in domestic human trafficking law. Boston University Law Review, 87, Retrieved from: http://128.197.26.3/law/central/jd/organizations/ journals/bulr/volume87n1/documents/SRIKANTIAHv.2.pdf

Tsutsumi, A., Izutsu, T., Poudyal, A. K., Kato, S., \& Maru, E. (2008). Mental health of female survivors of human trafficking in Nepal. Social Science \& Medicine, 66, 1841-1847.

United States Department of Justice [US DOJ]. (2006). Report on activities to combat Human trafficking: fiscal years 20012005 [electronic version]. Washington: U.S. Government Printing Office.

United States Department of State [US DOS]. (2006). Trafficking in persons report. Washington: U.S. Government Printing Office.

United States Department of State [US DOS]. (2007). Trafficking in persons report. Retrieved from: http://www.state.gov/documents/ organization/82902.pdf.

Zakrzewski, S., Steven, C., \& Ricketts, C. (2009). Evaluating computerbased assessment in a risk-based model. Assessment \& Evaluation in Higher Education, 34, 439-454.

Zimmerman, C. Hossain, M., Yun, K., Gajdadziev, V., Guzun, N., Ciarrocchi, R. A., Tchomarova, M., Johansson, Kefurtova, A., Scodanibblo, S., Nenette Motus, M., Roche, B., Morison, L., \& Watts, C., (2008). The health of trafficked women: A survey of women entering post-trafficking services in Europe. American Journal of Public Health, 98 